\title{
Electron Impact Single Ionization of the He-Isoelectronic Sequence
}

\author{
A. L. Frapiccini ${ }^{1,2}$, K. V. Rodriguez ${ }^{1}$, G. Gasaneo ${ }^{1}$, and S. Otranto ${ }^{1}$ \\ ${ }^{1}$ Departamento de Física, Universidad Nacional del Sur and CONICET, \\ 8000 Bahía Blanca, Buenos Aires, Argentina. \\ ${ }^{2}$ Centro Atómico Bariloche and CONICET, \\ 8400 Bariloche, Río Negro, Argentina.
}

Received on 12 April, 2007

\begin{abstract}
In this work, triply differential cross sections for single electron emission due to electron impact on the Heisoelectronic sequence are calculated by using a Born-C3 model. The influence of the nuclear charge on the angular distributions is analyzed. The validity of a scaling law initially derived in the framework of photodouble-ionization is discussed.
\end{abstract}

Keywords: Electron collisions; Single ionization; Fully differential cross sections; He-isoelectronic sequence

\section{INTRODUCTION}

The electron-impact single ionization of He has been subject of many studies during the last thirty five years. The techniques to measure Triply Differential Cross Sections (TDCS) were developed in the late 1960s [1, 2]. Since then, the improvement of these techniques systematically pushed theoreticians to improve the usual models employed in order to describe that problem.

From the theoretical point of view, the high energy models based on Born and eikonal-Born series were the standard in order to describe the collision process (see [3] for a review). By the end of the 1980s, Brauner et al. showed that the interelectronic interaction must be included in the final state wave function in order to correctly describe the angular distribution of the emitted electron for single ionization of hydrogen [4]. In that work the separable C3 model consisting in the product of three Coulomb wave functions, previously used in ion-atom collisions [5], was employed to represent the two electron continuum together with an uncorrelated Born initial state which mainly consists in a plane wave for the incoming projectile times a bound state for the target atom. This approximation clearly improved the description of angular distributions for electrons impinging with intermediate and high energies. This model was soon afterwards adapted to describe the single ionization of $\mathrm{He}$ [6], by representing the two electron continuum by means of the $\mathrm{C} 3$ model and considering an effective residual charge for the remaining ionic core. In this work, the $\mathrm{C} 3$ model correctly reproduces the experimental data for asymmetric geometries.

On the other hand, in the threshold region where the Wannier emission mechanism prevails [7], the C3 model fails by orders of magnitude to the absolute cross sections due to an overestimation of the interelectronic repulsion. This model, being a solution of an approximated Hamiltonian which neglects the kinetic-correlation given by the non-diagonal terms of the metric tensor, does not include any information on the screening of the electron-electron interaction by the ionic core. This leads to an exponential decreasing behavior for the cross sections, instead of the well known power law dependence arising from Wannier theories.
The evaluation of the different models in the Wannier region could be also performed through the study of the considered atom isoelectronic-sequence, since the threshold region is then extended to higher energies [8]. These studies could include the analysis of the possible scaling of the different cross sections. In 1994 Kornberg and Miraglia derived scaling laws for $n$-differential cross sections for photo-double-ionization (PDI) of the He-isoelectronic sequence which have been theoretically tested during the last ten years [9-11]. A few years ago, Stia et al. confirmed that the same scaling laws are also valid for single-ionization of the hydrogen-isoelectronic sequence by electron impact [12]. However, up to our knowledge, no-similar analysis has been performed for the He-like ions for different target nuclear masses.

During the last years, many other authors concentrated towards the improvement of the $\mathrm{C} 3$ wave function for the threebody continuum in the low energy limit. In some cases, C3like models based upon the introduction of momentum and coordinate-dependent Sommerfeld parameters in the standard C3 model proved to be useful in $(\mathrm{e}, 2 \mathrm{e})$ and $(\mathrm{e}, 3 \mathrm{e})$ processes together with Born initial states [13-17]. These analysis were based on the satisfaction of several spatial and energetic asymptotic limits, giving different behaviors for the wave functions in the usually denominated reaction region, where all the particles are close to each other. In many physical processes, the initial bound state modulates the contribution of the continuum wave functions to the transition amplitude, and a correct description of the reaction region turns vital. Only continuum-continuum transitions include information from the asymptotic regions where these models are supposed to be valid.

Analytical models based on several variables hypergeometric functions were also developed by asking the wave function the satisfaction of several desirable physical limits [18, 19]. However, these models which partially introduce the kineticcorrelation have been only applied to photo-double ionization of $\mathrm{He}$ [20] and up to date they have not been tested in single ionization of atoms by light particle impact.

By the other side, numerical treatments have also been developed for this process. The Convergent Close Coupling (CCC) method of Bray and Fursa [21] numerically solves the 
TABLE I: Variational parameters for the He-isoelectronic sequence according to Eq.(9)

\begin{tabular}{llllll} 
atom & $a$ & $b$ & $N_{i}$ & $\langle E\rangle$ & $\langle E\rangle_{\text {exact }}$ \\
\hline$H^{-}$ & 1.0394 & 0.2834 & 0.0315 & -0.5133 & -0.5277 \\
$H e$ & 2.1882 & 1.1882 & 0.7088 & -2.8757 & -2.9037 \\
$\mathrm{Li}^{+}$ & 3.2945 & 2.0795 & 2.9639 & -7.2487 & -7.2799 \\
$\mathrm{Be}^{2+}$ & 4.3896 & 2.9852 & 7.7556 & -13.6229 & -13.6555 \\
$B^{3+}$ & 5.4716 & 3.9029 & 16.0371 & -21.9975 & -22.0309 \\
$C^{4+}$ & 6.5525 & 4.8236 & 28.7717 & -32.3723 & -32.4062 \\
$N^{5+}$ & 7.6201 & 5.7549 & 46.8943 & -44.7471 & -44.7814 \\
$\mathrm{O}^{6+}$ & 8.6818 & 6.6918 & 71.3647 & -59.1219 & -59.1565 \\
$F^{7+}$ & 9.7432 & 7.6321 & 103.188 & -75.4969 & -75.5317
\end{tabular}

continuum by expanding the total wave function in an orthogonal Laguerre basis treating the target continuum via a squareintegrable representation. This method though not satisfying the correct asymptotic limits has been applied with success for the He target case.

In this work we evaluate the single ionization of the Heisoelectronic sequence by electron impact. In section 1, the theoretical background is presented. In section 2 , the angular distributions for the ionization of He-like ions are obtained and different effective charges for the remaining ionic core are proposed and evaluated. Results for different impact energies and scattering angles are presented and discussed. The validity of the scaling law initially derived by Kornberg and Miraglia in the PDI context is evaluated and discussed. Conclusions and outlooks are presented in section IV. Atomic units are used unless explicitly stated.

\section{THEORY}

The triply differential cross section for the electron impact ionization of Helium is given by

$$
\frac{d^{3} \sigma}{d \Omega_{1} d \Omega_{2} d E_{1}}=(2 \pi)^{4} \frac{k_{1} k_{2}}{k_{0}}\left(\frac{1}{4}\left|T_{f i}^{S}\right|^{2}+\frac{3}{4}\left|T_{f i}^{T}\right|^{2}\right)
$$

where the direct and exchange transition matrix are given by

$$
\begin{aligned}
T_{f i}^{S} & =\left(1+\mathcal{P}_{12}\right) T_{f i}\left(\mathbf{k}_{1}, \mathbf{k}_{2}\right) \\
T_{f i}^{T} & =\left(1-\mathcal{P}_{12}\right) T_{f i}\left(\mathbf{k}_{1}, \mathbf{k}_{2}\right)
\end{aligned}
$$

Here, $k_{1,2}$ represent the momenta of the outgoing projectile and the emitted electron respectively, $k_{0}$ the incoming projectile momentum and $\mathcal{P}_{12}$ the permutation operator.

The transition amplitude $T_{f i}$ in the two active electron representation in prior form is given by

$$
T_{f i}=\left\langle\Psi_{f}^{-}\left|W_{i}\right| \chi_{i}^{+}\right\rangle
$$

where $\Psi_{f}^{-}$represents the product of a wave function for the double continua for the scattered and ejected electrons times a wave function for the remaining bound electron. $\chi_{i}^{+}$is the initial state and $W_{i}$ is the perturbation given by the part of the Hamiltonian that is not solved by $\chi_{i}^{+}$. In the present analysis we use an uncorrelated Born initial state which consists on a plane wave for the incoming projectile times a bound state representing the $1 s^{2}\left({ }^{1} S\right)$ state for the ground state of the atom.

$$
\chi_{i}^{+}=(2 \pi)^{-3 / 2} e^{i \mathbf{k}_{0} \cdot \mathbf{r}_{1}} \Psi_{1 s^{2}}\left(\mathbf{r}_{2}, \mathbf{r}_{3}\right) .
$$

The perturbation is then given by,

$$
W_{i}=-\frac{Z_{T}}{r_{1}}+\frac{1}{r_{12}}+\frac{1}{r_{13}}
$$

It could be observed that the perturbation operator consists in the projectile-nucleus interaction, and the interaction of the incident projectile with the two active electrons in the bound state.

It should be noted that this is a high energy approximation and a more precise representation would include Coulomb or eikonal waves to represent the interaction between the impinging projectile and the target. For that reason, in the following we restrict ourselves to study high energy collisions involving low momentum transfers by the projectile. It has been already shown in Ref. [12] that even a much simpler model than the one here employed, like the First Born Approximation, provides in this regime almost identical results compared to more elaborated CDW models.

For the continuum state part of the final wave function we use the C3 model,

$$
\begin{gathered}
\Psi_{C 3}^{-}=\Psi_{1 s}\left(\mathbf{r}_{3}\right) \sqrt{2}(2 \pi)^{-3} N_{C 3}^{-} e^{i \mathbf{k}_{1} \cdot \mathbf{r}_{1}+i \mathbf{k}_{2} \cdot \mathbf{r}_{2}} \\
\quad \times \prod_{j=1,2,12}{ }_{1} F_{1}\left[i a_{j}, 1,-i k_{j} r_{j}-i \mathbf{k}_{j} \cdot \mathbf{r}_{j}\right],
\end{gathered}
$$

being $a_{j}=Z_{j} \mu_{j} / k_{j}$ with $j=1,2,12$ the Sommerfeld parameters, $Z_{j}$ the charges, $\mu_{j}$ the relative masses and $\mathbf{k}_{12}=$ $\left(\mathbf{k}_{1}-\mathbf{k}_{2}\right) / 2$. The $\sqrt{2}$ is included to compensate since the wave function is not symmetrized. The wave function $\Psi_{1 s}\left(\mathbf{r}_{3}\right)$ represents the remaining bound electron in the ion. The normalization factor obtained from the Redmond asymptotic condition in the $\Omega_{0}$ region where the three particles are far from each other is given by,

$$
N_{C 3}^{-}=\prod_{j=1,2,12} e^{-\frac{\pi}{2} a_{j}} \Gamma\left(1-i a_{j}\right) .
$$

For two electrons in the field of an ionic core, the Sommerfeld parameters are given by

$$
\begin{aligned}
& a_{1}=-\frac{Z_{e_{1}}}{k_{1}} \\
& a_{2}=-\frac{Z_{e_{2}}}{k_{2}} \\
& a_{12}=\frac{1}{2 k_{12}}
\end{aligned}
$$

where $Z_{e 1,2}$ refer to the ionic effective charge seen by the projectile and the emitted electron. 


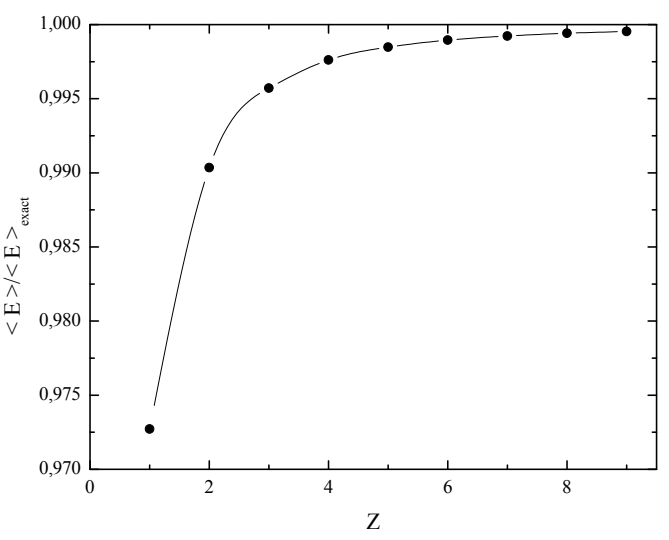

FIG. 1: Ratio of the variational to the exact binding energy as a function of the nuclear charge $Z$ for the wave function.

For the initial state, we use a simple Bonham and Kohl type wave function [22] which only includes radial correlation:

$$
\Psi_{1 s^{2}}\left(\mathbf{r}_{2}, \mathbf{r}_{3}\right)=N_{i}\left(e^{-a r_{1}-b r_{2}}+e^{-b r_{1}-a r_{2}}\right)
$$

where $a$ and $b$ are the variational parameters which are determined for different $Z$ charges as presented in Table I.

This wave function is supposed to be more precise as the nuclear charge increases. In Fig. 1 we present the ratio between the variational and the exact binding energies. As could be seen, for the lower charges the angular correlation seems to give a significant contribution but as the nuclear charge increases, the binding energy is almost recovered by just including radial correlation. This means that as the nuclear charge increases so does the nuclear field, turning the electrons insensitive to their relative positions. Then, the inclusion of the screening or radial correlation suffices to give a good description of the two-electron system.

\section{RESULTS}

According to the models introduced in the previous section, we can stress that the most important weakness of the present theory relays in the treatment of the ionic charges $Z_{e 1, e 2}$ which must be empirically included.

A more elaborate description would include the solution of an effective radial potential, as could be a Herman-Skillman potential based on the Hartree-Fock methodology. However, based on the good qualitative results obtained in the following analysis we represent screening effects through effective charges.

Since we restrict the present analysis to very asymmetric geometries, we now consider and evaluate two different models for the ionic charges: a) $Z_{e 1}=Z_{e 2}=\left(Z_{T}-1\right)$ and b) $Z_{e 1}=\left(Z_{T}-1\right), Z_{e 2}=\left(Z_{T}-5 / 16\right)$.

These models mainly differ in how the remaining bound electron after the collision screens the nuclear charge. In the first model, which could be considered a high energy limit, the

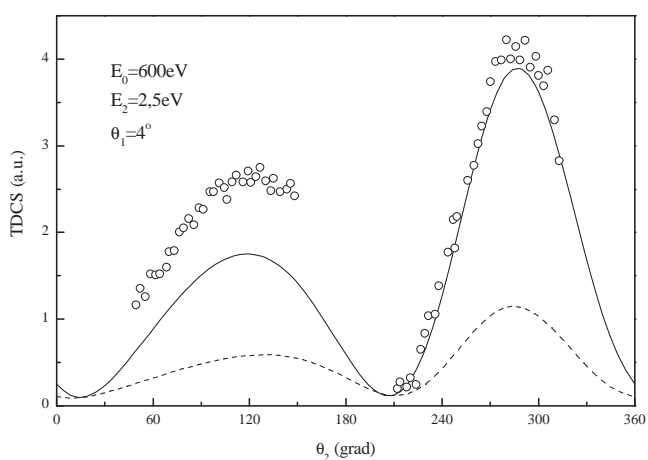

FIG. 2: TDCS under asymmetric geometry. The impinging energy is $600 \mathrm{eV}$ and the scattering angle considered is $4^{\circ}$. The emission energy is $2.5 \mathrm{eV}$. Theories: solid-line: $Z_{e 1, e 2}=Z_{T}-1$, dashed-line: $Z_{e 1}=Z_{T}-1$ and $Z_{e 2}=Z_{T}-5 / 16$.

scattered projectile and the emitted electron see the nuclear charge reduced in one unit. In other words, they see the maximum possible screening of the nuclear charge by the remaining bound electron. This model was already used by Brauner et al. [6] for electron impact ionization of $\mathrm{He}$ in the high energy limit under asymmetric configurations. As a result they obtained good qualitative agreement with experimental data. In the second model, the scattered projectile sees an effective charge $Z_{T}-1$, while the slowly emitted electron now sees a partial screening $Z_{T}-5 / 16$. As it was pointed out by Berakdar and Briggs [23], within the frame of an equal energy sharing analysis, it could be expected that the ionic charge seen by a fast receding particle should tend to $Z_{T}-1$ as its energy tends to infinity. According to this, they proposed a functional form for the core charge by fitting available experimental data. However different ways to approach the high energy limit could be considered. In this sense, the second model recovers part of this philosophy but makes no use of experimental data to fix the charges.

In Fig. 2, we present the TDCS under asymmetric energy conditions for electron impact ionization of He. The impinging energy is $600 \mathrm{eV}$, the scattering angle considered is $4^{\circ}$ and the emission energy considered is $2.5 \mathrm{eV}$. The absolute experimental data of Jung et al. [24] are included for comparison to the theoretical results. The two above proposed models are compared and as a result, we conclude that model $a$ is the one which better approximates the shape of the data. This could be explained by comparing the different partial waves obtained as a solution of the radial two particle Coulomb problem, including the presence of a second electron by a Herman $\&$ Skillman type potential. For emission energies lower than $5 \mathrm{eV}$, we found that only the $l=0$ partial wave is modified by the screening, the other partial waves being coincident with the Coulomb partial waves for $Z=1$. Then in the forthcoming analysis we restrict ourselves to model $a$.

In Figs. 3 and 4 we present the TDCS for two particular geometries and energies for different He-like ions. In Fig. 3, the impact energy of the light particles is $2 \mathrm{keV}$. The emitted electron energies are $2.5 \mathrm{eV}$ and $5 \mathrm{eV}$ and the scattering angle 

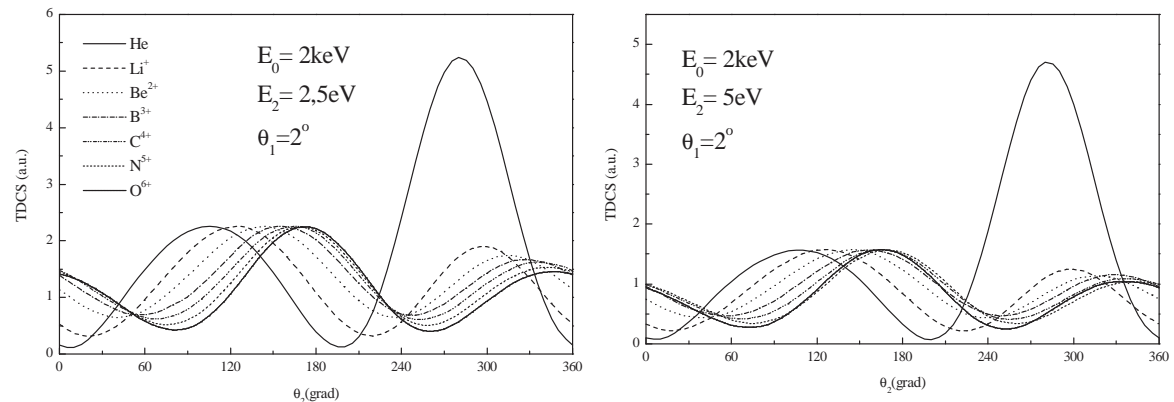

FIG. 3: TDCS in asymmetric geometry for an impact energy of $2 \mathrm{keV}$ for scattering angle $2^{\circ}$. Two different emission energies are considered: $2.5 \mathrm{eV}$ and $5 \mathrm{eV}$. Theories: solid-line: $\mathrm{He}$; dashed-line: $\mathrm{Li}^{+}$; dotted-line: $\mathrm{Be}^{2+}$; dot-dashed-line: $\mathrm{B}^{3+}$; dash-dot-dotted-line: $\mathrm{C}^{4+}$; short-dashedline: $N^{5+}$.
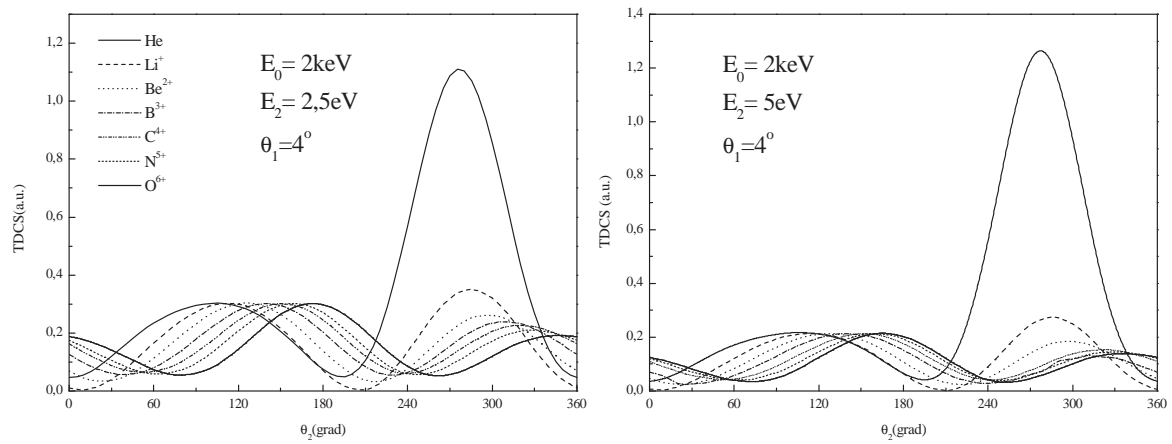

FIG. 4: Same as Fig. 3 for a scattering angle of $4^{\circ}$. Theories: solid-line: $H e$; dashed-line: $\mathrm{Li}^{+}$; dotted-line: $B e^{2+}$; dot-dashed-line: $B^{3+}$; dash-dot-dotted-line: $\mathrm{C}^{4+}$; short-dashed-line: $\mathrm{N}^{5+}$; short-dotted-line: $\mathrm{O}^{6+}$.

is $2^{\circ}$. All the curves have been scaled to the He case and we have normalized the results to the recoil peak maximum value. In both situations, we can see that the double collision mechanism becomes dominant as the nuclear charge increases. This means that ionization through a single collision mechanism between the projectile and the emitted electron is much less probable than a two step mechanism, where the electron is first hit by the projectile and then is scattered by the nucleus. It could also be observed a significant shift in the positions of both peaks. This could be attributed to the fact that for high $\mathrm{Z}$-values, the bound electrons are closer to the nucleus and so the ionization is more similar to the large impact parameter. It is less probable for the projectile to produce a"head-on" collision because the atom is smaller. Then, the large impact parameter situation is enhanced just because a simple reduction of the atomic size occurs. It is also well known that high impact parameter collisions occur mostly through the momentum exchange between the emitted electron and the nucleus, explaining why the recoil peak turns to be the dominant structure in the spectrum. In Fig. 4 a scattering angle of $4^{\circ}$ is considered. The impact energy and emitted electron energies are the same as in Fig. 3. Similar trends can be observed, which give clear footprints of the enhancement of the contribution to the cross sections arising from double-collisions.

In 1994, Kornberg and Miraglia [9] derived a scaling law for the nuclear charge dependence of the PDI cross sections.

$$
\begin{gathered}
\sigma_{\text {tot }}\left(E_{f}, Z_{T}\right) \rightarrow \frac{1}{Z_{T}^{4}} \sigma_{\text {tot }}\left(E_{f} / Z_{T}^{2}, Z_{T}=1\right) \\
\frac{d \sigma}{d E_{1}} \rightarrow \frac{1}{Z_{T}^{6}} \frac{d \sigma}{d\left(E_{1} / Z_{T}^{2}\right)}\left(E_{f} / Z_{T}^{2}, Z_{T}=1\right) \\
\frac{d^{5} \sigma}{d \Omega_{1} d \Omega_{2} d E_{1}} \rightarrow \frac{1}{Z_{T}^{6}} \frac{d^{5} \sigma}{d \Omega_{1} d \Omega_{2} d\left(E_{1} / Z_{T}^{2}\right)}\left(E_{f} / Z_{T}^{2}, Z_{T}=1\right)
\end{gathered}
$$

These scaling laws were recently confirmed for electron impact ionization of hydrogen-like ions [12]. In the present analysis we evaluate the TDCS, and check the validity of the above presented scaling to the He-isoelectronic sequence. In other words, we evaluate if $Z_{T}^{6} d^{5} \sigma /\left(d \Omega_{1} d \Omega_{2} d\left(E_{1} / Z_{T}^{2}\right)\right)$ tends to a uniform angular distribution for increasing $Z_{T}$. In Fig. 5 we present the scaled TDCS as a function of the emitted electron angle $\theta_{2}$. It could be observed that the scaling is meaningful even in this situation in which the remaining core consists on a dressed ion. It could be also noted that for high values of $Z_{T}$ (and equivalent energies) the recoil peak 

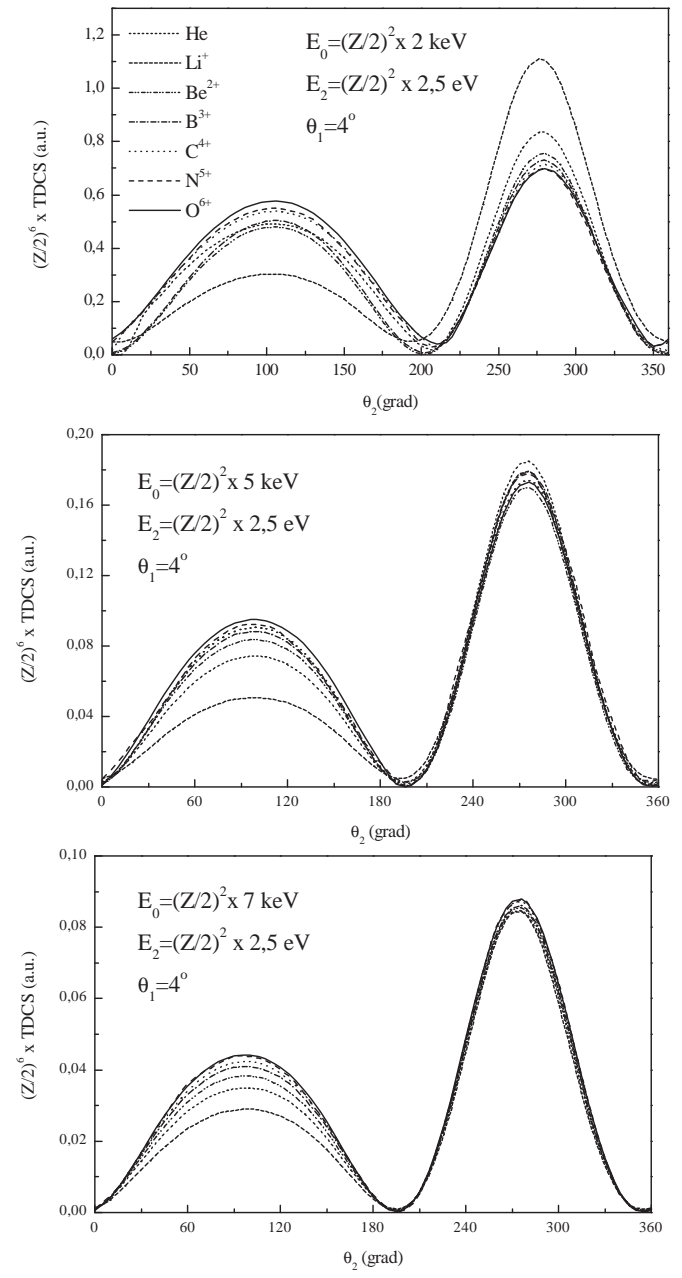

FIG. 5: Scaled TDCS corresponding to impact energies $2 \mathrm{keV}, 5 \mathrm{keV}$ and $7 \mathrm{keV}$ for $\mathrm{He}$ and scattering angle $4^{\circ}$. Theories: dash-dot-dottedline: $\mathrm{He}$; short-dotted-line: $\mathrm{Li}^{+}$; short-dashed-line: $\mathrm{Be}^{2+}$; dotdashed-line: $B^{3+}$; dotted-line: $C^{4+}$, dashed-line: $N^{5+}$, solid-line: $\mathrm{O}^{6+}$.

is more prominent when compared with the He target case. This feature could be explained as follows. It is well known from single ionization of He under asymmetric geometries, that the recoil peak intensity decreases as the projectile energy increases or as the projectile wavelength decreases compared to the target size.

For high charges, the two-electron system is quasiseparable and the energy is proportional to the second power of the charge. The scaled Schrödinger equation leads to a scaled momenta (wavelength) which behaves as $Z_{T}\left(1 / Z_{T}\right)$. Thus, according with the independent particle model the size of the atom and the wavelength of the electrons in the continuum scale with the same law. As consequence, the angular distributions should not be altered when the nuclear charge is modified. However, in the real two-electron system an increasingly significant departure from the independent particle model is observed when the nuclear charge is lowered towards the He limit. In that situation, the electron-electron correlation keeps the atomic size bigger than the one resulting from the independent particle model. The mentioned difference then recreates a high energy situation where the recoil peak reduces its size compared to the binary one. By the other side, as the nuclear charge increases, the atomic size to projectile wavelength ratio decreases and tends to a constant, fact which leads to the convergence of the scaling.

\section{CONCLUSIONS AND OUTLOOKS}

In this work, we have presented TDCS for the ionization of two electron ions by electron impact. We have used a Born-C3 model which entirely considers in the final state the Coulomb interactions between the scattered projectile, the emitted electron and the remaining ionic core.

The influence of the nuclear charge on the angular distributions obtained at fixed energetic and angular configurations has been analyzed and explained considering the relation between physical emission mechanisms and the respective atomic size. From our calculations, a more intense recoil peak has been obtained in all cases as the nuclear charge considered was increased. This has been attributed to the fact that the simple reduction of the atomic size enhances the relevance of large impact parameter collisions which mainly occur through the momentum exchange between the emitted electron and the nucleus.

We have shown that the Kornberg and Miraglia scaling laws, already proved in $(\gamma, 2 e)$ and $(e, 2 e)$ processes consisting in pure three body final states, are also valid in the context of dressed remaining cores. This would imply that these scaling laws are related to the number of electrons which are in the continuum, but not to the internal structure of the considered atom. Further investigations are being addressed in that direction.

Although up to date there is no data available for this type of process, we believe the present analysis could be of interest as soon as trapping of ions would become routine. When the nuclear charge considered increases, and in particular for low energy values, a wide window of the threshold region is obtained as recently observed in PDI of the He-isoelectronic sequence [8]. This could be important to gain insight in the Wannier region and the validity of the different semiclassical models that have been proposed during the last thirty years.

This work has been supported by PICT 03/0437 of the ANPCyT, PIP 5595 of CONICET and PGI 24/F038 UNS (Argentina).
[1] U. Amaldi Jr, A. Egidi, R. Marcoreno, and G. Pizzella, Rev. Sci. Instr. 40, 1001 (1969).
[2] H. Ehrhardt, M. Schulz, T. Tekaat, and K. Willmann, Phys. 
Rev. Lett. 22, 89 (1969).

[3] F. W. Byron Jr and C. J. Joachain, Phys. Rep. 179, 211 (1989).

[4] M. Brauner, J. S. Briggs, and H. Klar, J. Phys. B: At. Mol. Opt. Phys. 22, 2265 (1989).

[5] C. R. Garibotti and J. E. Miraglia, Phys. Rev. A 21, 572 (1980).

[6] M. Brauner, J. S. Briggs, and J. T. Broad, J. Phys. B: At. Mol. Opt. Phys. 24, 287 (1991)

[7] G. H. Wannier, Phys. Rev. 90, 817 (1953).

[8] S. Otranto and C. R. Garibotti, Phys. Rev. A 71, 034703 (2005).

[9] M. A. Kornberg and J. E. Miraglia, Phys. Rev. A 49, 5120 (1994).

[10] A. S. Kheifets and I. Bray, Phys. Rev. A 58, 4501 (1998).

[11] S. Otranto and C. R. Garibotti, Eur. Phys. J. D 27, 215 (2003).

[12] C. R. Stia, O. A. Fojon, and R. D. Rivarola, J. Phys. B: At. Mol. Opt. Phys. 33, 1211 (2000).

[13] E. O. Alt and A. M. Mukhamedhanov, Phys. Rev. A 47, 2004 (1993).

[14] J. Berakdar and J. S. Briggs, Phys. Rev. Lett. 72, 3799 (1994).
[15] J. Berakdar, Phys. Rev. A 53, 2314 (1996).

[16] Z. Chen, Q. Shi, S. Zhang, J. Chen, and K. Xu, Phys. Rev. A 53, R2514 (1997).

[17] J. R. Götz, M. Walter, and J. S. Briggs, J. Phys. B: At. Mol. Opt. Phys. 38, 1569 (2005).

[18] P. A. Macri, J. E. Miraglia, C. R. Garibotti, F. D. Colavecchia, and G. Gasaneo, Phys. Rev. 55, 3518 (1997).

[19] J. E. Miraglia, M. G. Bustamante, and P. A. Macri, Phys. Rev. A 60, 4532 (1999).

[20] S. Otranto and C. R. Garibotti, Phys. Rev. A 67, 064701 (2003).

[21] I. Bray and D. V. Fursa, Phys. Rev. A 54, 2991 (1996); Phys. Rev. Lett. 76, 2674 (1996).

[22] R. A. Bonham and D. A. Kohl, J. Chem. Phys. 45, 2471 (1966).

[23] J. Berakdar and J. S. Briggs, J. Phys. B: At. Mol. Opt. Phys. 27, 4271 (1994).

[24] K. Jung, R. Muller-Fiedler, P. Schlemmer, H. Ehrhardt, and H. Klar, J. Phys. B: At. Mol. Opt. Phys. 18, 2955 (1985). 